\title{
REVIEW
}

\section{Neurodegenerative diseases and neuroprotection: current views and prospects}

\author{
André Nieoullon \\ Institut de Biologie du Développement de Marseille-Luminy (IBDML) CNRS, Aix-Marseille Université, \\ Marseille, France
}

Received $20^{\text {th }}$ July 2011.

Revised $16^{\text {th }}$ August 2011.

Published online $9^{\text {th }}$ September 2011.

\begin{abstract}
Summary
Most of the pathophysiological processes of neurodegenerative diseases share the aggregation of related proteins which is one of the hallmarks of the degenerative processes. Recent advances in the knowledge of these proteinopathies show that the same protein could contribute to a number of diseases, thus suggesting a common pathological process. If this is so, specific instances of the brain neuronal system targeted by protein dysfunction could be a sign of a differential clinical expression rather than different pathological processes. This very stimulating view of the neurodegenerative diseases based on physiopathology has led us to suggest that possible degenerative mechanisms may be shared by different diseases although the causes of the disease itself still remain unclear. Since genetic forms of the degenerative diseases are rather rare, exploring the involvement of genes is one current way of researching the degenerative process of the disease. It is has thus been speculated that idiopathic forms of the diseases are related to close interactions between genetic and environmental factors; the genetic component being able to favour - or perhaps, to protect against - the disease process. Because of the current view that the basic mechanism of cell death in degenerative diseases is related to a rather limited number of processes in which oxidative stress could play a central role resulting in protein dysfunction and aggregation, one can speculate that there are neuroprotective medicines soon to be proposed based on the active limitation of protein accumulation in the brain.
\end{abstract}

Key words: neurodegenerative diseases; proteinopathies; Alzheimer's disease; Parkinson's disease; oxidative stress; proteasome; neuroprotection

\section{INTRODUCTION}

Although Alzheimer's disease is in the forefront of our thinking about neurodegenerative disorders it should not be forgotten that such diseases in general form a very large proportion of the total number of

\footnotetext{
$\square \quad$ André Nieoullon, Institut de Biologie du Développement de Marseille-Luminy (IBDML) CNRS, Aix-Marseille Université, UMR6216, 13288 Marseille cedex 9, France$$
\text { 品 andre.nieoullon@univmed.fr }
$$$$
\text { 留 } 33491269240
$$

neurological diseases, and represent a very high financial cost for society. A recent study (see Andlin-Sobocki et al. 2005) has evaluated the total cost of brain diseases per year, including direct and indirect costs, in 28 countries in Europe at about 386 billion Euros for the year 2004. This represented $35 \%$ of the total burden of diseases affecting about $27 \%$ of the 465 million people who are suffering brain diseases. If mental disorders are excluded from the calculation the total cost of neurological diseases including dementia could be about 146 billion Euros per year and the total specific cost of the neurodegenerative diseases could be as much as 72 billion Euros (Table 1). These diseases are found in about $5 \%$ of the total number of patients suffering 
brain diseases. They are characterized by more or less selective neuronal degenerations inducing neurological syndromes, and affect both sensory-motor areas and cognitive functions.

\section{FROM NEURODEGENERATIVE DISEASES TO PROTEINOPATHIES}

Clinical semiology has contributed to the well accepted classification of the neurodegenerative diseases, the main representatives of which are Alzheimer's disease and related dementia, Parkinson's disease, amyotrophic lateral sclerosis (ALS), fronto-temporal-dementia (FTD) and Huntington's disease among many others. Recent advances in genetics and molecular biology have led us, however, to reconsider this clinical classification. The presence of amyloid peptide accumulations in the extracellular space, is considered a pathophysiological hallmark of Alzheimer's disease but such indications in general have been recently revisited, to take into account the fact that some of these diseases are characterized by similar intraneuronal accumulations of particular proteins such as for example the tau protein (acting as a microtubule associated protein). Such a special tau protein accumulation thus contributes to the delineation of a subgroup of neurodegenerative diseases considered as tauopathies, including Alzheimer's disease, cortico-basal degeneration, supra nuclear progressive paralysis and certain forms of FTD. Similarly, accumulations of $\alpha$-synuclein, where the natural function of the protein is still unknown, contribute to define the synucleinopathies subgroup, which includes Parkinson's disease, multisystematized atrophy and Lewy body dementia (Ozawa et al. 2006). More recently a subcategory of FTD was shown positive for another protein ubiquitin - but negative for tau protein accumulation. These particular FTD, however, accumulate the transcription factor TAR DNA-binding protein 43 (TDP-43), contributing to the definition of a new class of proteinopathies including FTD associated to ALS (Neumann et al. 2006, Josephs 2008).

These proposals (see Derkinderen 2009) are of major importance, since, although they do not change the clinical classifications, they are of considerable heuristic value and contribute to the idea of possible common pathophysiological processes, having very different clinical expressions. In short, in considering the similar conceptual approaches to these different diseases one can speculate on the actual possibility of future putative innovative treatments based on the etiology of diseases which may apply to the entire group of pathologies belonging to the same protein entity (see Vajda 2002).

\section{SOME COMMON CHARACTERISTICS OF THE NEURODEGENERATIVE DISEASES}

Neurodegenerative diseases have in common the very progressivity of neuronal death, and this contributes to the difficulty of establishing valuable animal models of the diseases. In general, such models, developed primarily using lesion experiments, do not in fact reproduce the progressivity of the disease. Unfortunately, the problem is not solved by genetic modelling of the diseases based on selected gene inactivation or over-expression in mice. Thus, the pathophysiological mechanisms of the diseases in general still remain largely unknown.

The progressivity of neuronal death has also led to speculation on the very long prodromal phase of the diseases, which asks key questions concerning early diagnosis and treatment; a stage at which the clinical signs of the disease are not expressed. The existence of a prodromal phase of the diseases also contributes to questions about mechanisms of the brain related to neuroplasticity, which very efficiently compensate possibly for decades - for the clinical expression of related brain lesions. It is thus claimed that the earlier the treatment of the disease, the better it would be for limitation of neuronal destruction and clinical expression. Such a consideration emphasizes the search for early events of the diseases and critical putative biomarkers (see Hampel et al. 2010) as well as stimulating research programs to promote therapeutical approaches focused at the etiological level leading to true neuroprotection against the diseases.

In addition to the ethical problems linked to the announcement to the patient of a heavy diagnosis in the absence of curative treatment - such as for example in familiar diseases of the Huntington's disease type - the question of early diagnosis also points to the difficulty clinicians have in relation to differential diagnosis at a stage when clinical signs are still limited. Such early diagnosis, however, is absolutely necessary to further develop the evaluation of the rather subtle first putative clinical signs of the disease. Clinical evaluation and development of cerebral brain imaging methods would further contribute to early diagnosis of the neurodegenerative diseases. Interestingly such early diagnosis also involves the development of new biomarkers, the validation of which is currently carried out to produce an index of the follow up of developing brain lesions 
Nieoullon: Neurodegenerative diseases and neuroprotection

Table 1. Number of cases and cost per case and per year of neurodegenerative diseases in 28 selected European countries (2004)*

\begin{tabular}{lccc}
\hline & Number of cases & Cost per case/year & Total cost/year \\
\hline Parkinson's disease & 1160000 & 7500 Euros & 8.70 billion Euros \\
Dementia & 4890000 & 11000 Euros & 53.80 billion Euros \\
Multiple sclerosis & 380000 & 24000 Euros & 9.12 billion Euros \\
\hline
\end{tabular}

* from Andlin-Sobocki et al. (2005)

(Hampel et al. 2010). For example, in Alzheimer's disease new measurements of CSF concentrations of tau protein (native and/or phosphorylated forms) and/or A $\beta 1-42$ peptide are still possible, although certain of these biomarkers could also be linked to some other forms of the neurological diseases, which question their actual specificity.

Another current characteristic of the neurodegenerative diseases is linked to the rather selective systemic aspect of brain lesions, at least at the time when the degenerative process is starting. Such selectivity asks for the determination of the conditions of the preferential vulnerability of the given neuronal systems compared to others, and further suggests possible therapeutic responses oriented toward these particular neuronal systems when such conditions will be determined. This question also constitutes an open field for further investigation in the study of the neurodegenerative diseases.

\section{WHAT ABOUT THE CAUSES OF NEURODEGENERATIVE DISEASES?}

In the sporadic forms of the neurodegenerative diseases, causative factors still remain largely unknown. For Alzheimer's disease and possibly Parkinson's disease, ageing is considered as a major risk factor because of the increased incidence of the diseases with age. In the case of Alzheimer's disease the situation is clear, but in the other case such an assessment is open to question since for a large proportion of patients the starting point of Parkinson's disease could be very early, and masked by a long prodromic phase of the disease.

Some familiar forms of the diseases reveal a genetic component but monogenic cases are very rare and even exceptional (see Corti et al. 2010). This is the case in early-onset forms of Alzheimer's disease where the amyloid hypothesis is supported by mutations of genes causing the disease, such as mutations of gene encoding amyloid protein precursor (chromosome 21) or presenilins (PS1 and PS2 located on chromosomes 1 and 14 , respectively) in rare autosomic dominant forms $(<5 \%)$. A similar situation is found, for example, in autosomic dominant forms of Parkinson's disease, with the gene encoding $\alpha$ synuclein (mutation PARK1 and PARK4) and LRRK2 (mutation $P A R K 8$ ) among 15 different identified mutations. In some families, where FTD and ALS cosegregate, mutations on one gene linked to chromosome 19 could induce both clinical phenotypes, which supports the concept of a common mechanism, as revealed by new data on protein accumulations (see Sleegers et al. 2010).

On the other hand, it has been speculated that, in some patients, environmental factors can be involved in the development of particular neurodegenerative diseases (Cicchetti et al. 2009). For example, compounds such as fungicides, pesticides, heavy metals, substances derived from some addictives drugs, or even a virus, could possibly play a role in causing Parkinson's disease (see Moisan et al. 2011). However epidemiologic studies have to be further developed since present conclusions are in general not sufficiently clear, therefore suggesting that sole exposures to such environmental factors - even when prolonged and intensive - are not usually sufficient to induce the diseases (Dick 2006).

Interestingly, in relation to environmental factors which could influence the occurrence of neurodegenerative diseases, a new direction has recently been taken focusing on stress. Intensive chronic stress in humans was for example associated with reduced hippocampal and right orbito-frontal volumes (Gianaros et al. 2007), which may impact cognitive and emotional functions. Consequently, the 
durable altered activity of the hypothalamo-pituitary axis could affect brain integrity. In experimental studies, however, the most potent effects of stress are observed when experiences occur in the early or even prenatal phases of life. Indeed, stress in early life appears to induce changes in adult behavior, such as increased anxiety (see Marais et al. 2008). Experiments using early maternal separation in rodents show reduced hippocampal volume (Llorente et al. 2009) and thickness of the dorsal prefrontal cortex (Spivey et al. 2009), concomitant with increased neuronal apoptosis in the hippocampus and cortex. It has therefore been proposed that maternal stress sensitizes the individual to later stress factors. Thus, in the case of Parkinson's disease, it was speculated that pathogenic events occurring during early development may actually represent a source of vulnerability to the later disease (see Barlow et al. 2007). In this respect maternal separation in rats was shown to increase 6-hydroxydopamine-induced behavioural impairment in the adult (Mabandla and Russell 2010). So, early chronic stress could possibly be a factor of vulnerability to Parkinson's disease and possibly more generally of neurodegenerative diseases in the adult, the mechanisms of which have to be further investigated.

So, it is generally admitted that the sporadic cases of neurodegenerative diseases - the vast majority in fact - are linked to close critical interactions between environmental and genetic factors. Consequently, it is proposed that the differential expression of certain genes or selected gene mutations will predispose to the toxic action of environmental factors on specific neuronal populations. Such a genetic susceptibility to environmental factors would explain why certain people develop the disease whereas others in the same population do not when subjected to similar environmental conditions. In the same way it can be proposed that if certain people are more sensitive than others to environmental conditions, then conversely the apparent resistance to such factors could be linked to specific genetic "neuroprotective" factors. There is presently a lack of direct evidence for this, but we know that the differential expression of $\varepsilon 2, \varepsilon 3$ or $\varepsilon 4$ alleles of the apolipoprotein E ( $A P O E$ ) gene located on chromosome 19 could either increase or decrease the risk factor of developing Alzheimer's disease.

Indeed, in the CNS, APOE is primarily synthesized and secreted by astrocytes and microglial cells and contributes to the metabolism of cholesterol and lipids. Experimentally the astrocytic expression of human $A P O E 3$ or $E 4$ has resulted in an isoform-dependent effect on the $A \beta$ peptide deposit thus showing increased deposits in the case of $A P O E 4$ expression (Holtzman et al. 2000). Thus APOE4 appears as a true genetic risk factor since its frequency increases in late-onset forms of Alzheimer's disease. Conversely, the $\varepsilon 2$ isoform may represent a factor contributing to the reduction of the risk of developing the disease by acting as a "neuroprotective". In this context the over-expression of the $\varepsilon 4$ allele is a risk factor possibly interacting with other genetic or non genetic factors to facilitate the occurrence of Alzheimer's disease.

Other theories have been developed to explain the occurrence of neurodegenerative diseases. Briefly, 3 groups of factors have been proposed. The first group of theories involves endogenous factors inducing a neuronal toxicity such as free radicals at the origin of oxidative stress or the neurotransmitter glutamate, which could destroy neurons by a process called "excitotoxicity". The second group of theories assumes that the neurodegenerative process could be due to a lack of production or the action of more or less specific energetic (mitochondrial defect) or neurotrophic cellular factors; an impairment of physiological retrograde axonal transport; an alteration in cellular adhesion processes, or even to a synaptic deficit in the neurotransmitter function or synaptic metabolism such as glutamate inactivation mechanisms. Finally, neurodegenerative processes could also be linked to immunodeficiency mechanisms such as in the case of auto-immune pathologies which involve specific proteins exposed to cellular surface, or to inflammatory processes. Inflammatory processes, possibly involving microglial cells are indeed presently thought to be of primary importance in neurodegeneration such as in Parkinson's disease (see Hirsch and Hunot 2009). Last but not least, some other hypotheses of the abiotrophic family have been proposed, suggesting that neuronal degeneration involves an increase of natural cellular death mechanisms or, in some cases, alterations of the cell cycle.

It is therefore worth mentioning that if neurodegenerative processes can be linking to such different mechanisms, involving either/or genetic components and external/internal signals, the mechanisms of neuronal death could, conversely, involve a considerably limited number of cellular and/or molecular events. These events could represent key elements as common effectors of cellular death in neurodegenerative diseases. Such a consideration thus refers to the above presented classification of proteinopathies which may involve for each of them several neurodegenerative processes suggesting that putative common therapeutic solutions have to be considered. 


\section{FURTHER CONSIDERATIONS ON NEURONAL DEATH MECHANISMS}

It is worth mentioning at the outset, that nowadays, in spite of exceptional advances in cellular and molecular biology, it is quite impossible to precisely evaluate the contribution of apoptosis mechanisms in neurodegenerative diseases. We can assume, however, that such a mechanism is involved. Apoptosis refers to an active process of cellular death involving selective activation of a family of cystein-proteases and associated proteins such as for example the PARP protein, a protein polymerase involved in DNA reparation processes, and numerous other proteins such as those corresponding to the caspase family (13 genes in human). Apoptosis, therefore, can be viewed, at least partly, as an organized and regulated cascade of gene activation encoding cystein-proteases, the final issue of which is endonuclease activation, inducing characteristic structural changes in the cells. The apoptosis cascade, however, was primarily described from physiological data related to developmental processes contributing to the elimination of over-numeric cells, which depend, in the case of neurons, on cellular activity and the sufficient availability of specific trophic factors, and not from pathological processes. Nevertheless, it can be mentioned that either the prion protein or $\beta$-amyloid peptide are active in the promotion in vitro of DNA fragmentation similar to that seen in a true apoptosis mechanism. Such experimental data further suggest an involvement of apoptosis in neuronal death related to Creutzfeld-Jakob or Alzheimer's disease.

To suggest that apoptosis contributes as a sort of "final common pathway" to neuronal death in many neurodegenerative processes is consequently of great heuristic value since apoptosis is subjected to numerous genetic and epigenetic mechanisms including environmental factors. From such a point of view it is possible to imagine the intervention of "apoptosis-inducing factors" which can be opposed to the view of "apoptosis-inhibiting factors" which may contribute to tumourisation and cancers. Further, we can speculate that neuronal death in neurodegenerative diseases could actually be regulated. A strategy could be proposed to promote a true neuroprotection procedure balancing the neurodegenerative process and contributing to slowing and further ideally stopping the degenerative disease.

As already mentioned, free radical production is considered a main actor in the case of neuronal death in relation to the oxidative stress theory. Numerous experimental data support such a hypothesis. Indeed, the reduction of molecular oxygen is a source of superoxide derivatives, which is amplified by the production of nitric oxide (NO) or calcium ions contributing to deleterious cellular actions through proteases, endonucleases and calpains activation. Iron tissular concentrations as $\mathrm{Fe}^{2+}$ in combination with oxygen peroxide further contribute to the production of free radicals of the hydroxyl type (called the 'Fenton reaction') and may be involved in the degenerative process of dopamine neurons in the case of Parkinson's disease, because of the pro-oxidative state of the substantia nigra pars compacta where the neurons are located.

With reference to Parkinson's disease, it is worth mentioning that oxidative stress and free radical action are related to the increased activity of the microglial cells present in the neuronal environment, which could contribute to the promotion of oxidative stress through an increase in the production of cytokines and NO-synthase activation at the origin of an inflammatory state. This is the focus of the work of numerous research groups. In this respect the recent identification of a lymphocyte penetration into the substantia nigra studied in Parkinsonian patients (see Brochard et al. 2009) further reinforces this proposal and contributes to the justification for the use of anti-inflammatory medications in Parkinson's disease.

Oxidative stress can also be related to mitochondrial functional impairment, possibly inducing a decrease in complex I chain activity and cellular metabolism (Kristian et al. 2011). Consequently, oxidative phosphorylation could be reduced, membrane potential of the mitochondria will be lost and cytochrome $\mathrm{C}$ released into the cytoplasm, thus further contributing to caspase activation. Such a mitochondrial alteration could actually be involved in the development of certain neurodegenerative processes (Barsukova et al. 2011). For example, at least 3 of the mutated proteins described in Parkinson's disease (Parkin, DJ-1, and PINK1) are located in the mitochondria, which indicates a possible protective physiological role for mitochondria against the establishment of the disease.

In recent years, research has also been focused on the protein degradation process involving proteasome. Such a process could represent another major possible explanation for some degenerative diseases. Indeed, ubiquitinylation is a known signal of protein degradation through the proteasome machinery. Such a process also depends on ATP and request sequential activation of enzymes adding ubiquitin to substrate proteins (Berger et al. 2006). In this case, enzymes involved in desubiquitinylation processes (hydrolases) further contribute to ubiquitin recycling, 
which is a key step for addressing proteins to proteasome degradation machinery (Ardley et al. 2004). Events contributing to the degradation of proteins through proteasome activation are highly sensitive to oxidative stress and free radicals. In Parkinson's disease, for example, one of the enzymes contributing to the cycle known as ubiquitin ligase 3 is assimilated to the parkin gene, the mutation of which (PARK2 mutation) is associated with juvenile Parkinson's disease (recessive autosomal transmission). Similarly, the PARK8 mutation (dominant autosomal transmission) impairs leucin-rich repeat kinase (LRRK2; also called dardarin) protein function and could impair protein degradation through proteasome machinery and contribute to $\alpha$-synuclein deposits contributing indirectly to the formation of Lewy bodies in Parkinson's disease and, possibly, to hyperphosphorylated tau protein aggregates in Alzheimer's disease (see Abou-Sleiman et al. 2006, Berger et al. 2010).

Altogether these processes could be interdependent. One can speculate that it is possible to link in the same theory mitochondrial deficits, oxidative stress and proteasome machinery alterations, and even ubiquitin processing. Consequently, if one of these pathways is altered, such a limited defect will impair the others. In such conditions apoptose activation will actually be the final and definitive consequence for the cell.

\section{THE NEUROPROTECTION PARADIGM IN NEURODEGENERATIVE DISEASES}

In the context of the neurodegenerative diseases it is speculated that the administration of an etiopathogenic treatment which interferes with the developing pathogenic process, is able to slow and ultimately stop the evolution of the disease (see Djaldetti et al. 2003). Nowadays such a treatment for neurodegenerative diseases is not likely to exist in spite of the incredible number of attempts made in this direction (Velly et al. 2003, Labrande et al. 2006). Clinical trials with many hundreds of different compounds have been unsuccessful. Causes of such failures are multiple but at the same time it is highly paradoxical to consider that, conversely, preclinical data are extremely encouraging. Such a difference in trials could be primarily due to the unsatisfactory character of animal models of the neurodegenerative pathologies, which reflect only very partially the clinical situation. Moreover, such discrepancies could also be due to the actual difficulty clinicians have in evaluating neuroprotective effects in general because of very long lasting pathologies (see Akwa et al. 2005).

Although not fully satisfactory, the characterization of the mechanisms of neuronal death at cellular and molecular levels has nevertheless led to the proposition of new therapeutical strategies involving putative neuroprotective agents able to interfere and slow experimentally-induced neuronal death. In such a way the neuroprotection procedure aims at slowing the rate of neuronal death through interaction with the pathological process and furthering the evolution of clinical signs of the disease. The concept of a disease modifier was then introduced in this way, based on clinical improvement in patients. At the moment the concept of a disease modifier is more likely to be based on a mechanistic approach to the disease considered at cellular and molecular level than on a true neuroprotection process (see Citron 2004, Akwa et al. 2005).

If the different putative mechanisms of neuronal death here discussed are considered, one can speculate that the most popular approach to neuroprotection nowadays would be to try to reduce the oxidative stress at cellular level using anti-oxidant substances. In such a way numerous compounds have been proposed (vitamins A, C, E, polyphenols such as resveratrol or even baicalin or baicalein, Q co-enzyme, etc.) but clinical trials do not show any real improvement. Other strategies are based on oestrogen supplementation (see Tiwari-Woodruff et al. 2007), administration of anti-inflammatory drugs, calcium-stabilizing compounds or stimulators of the energy production of the cell. Altogether these compounds, directly or indirectly are thought to decrease the free radical oxygen species and thus oxidative stress. Interestingly, dietary restriction, phytochemicals (curcumin derivatives; flavonoids) and dietary lipids are also thought to act in the same way.

Further, agonism of the peroxisome proliferator receptor-gamma (PPAR) may have therapeutic interest for limiting oxidative stress and neuroinflammation in Parkinson's disease (Hunter and Bing 2007), where evidence has been found of an inflammation process in the substantia nigra of patients, thus corroborating data in animal models which further emphasize the contribution of glial cells and peripheral immune cells. Consequently downregulating these inflammatory processes could be of major importance for slowing the degenerative mechanisms (see Hirsch and Hunot 2009).

Ultimately, since genetic studies of the ageing process in animal models - primarily drosophila or the nematode Coenorhabditis elegans - have 
produced evidence of different sets of mutations related to an increased life-span for these animals, it can be speculated that inducing such gene mutations could contribute to increasing the resistance to diseases in general and to neurodegenerative diseases in particular. Interestingly it is worth mentioning that most of these positive mutations (age-1, daf-2, clk-1, old-1, etc.) result in decreasing oxidative stress and/or slowing cell metabolism (Friedman and Johnson 1988). For example, age- 1 encodes for a repressive factor of superoxide dismutase (SOD) expression and consequently the gene mutation results in increased resistance against the reactive oxygen species. Thus, it can be speculated that, in future, inducing such mutations in homolog human genes is a way to increase the resistance to the neurodegenerative diseases related to oxidative stress.

Apoptose inhibitors such as Bcl2 proteins, compounds acting on caspases, etc. have also been thought to reduce the impact of neurodegenerative diseases. Putative strategies could also involve modulation of NF- $\mathrm{kB}$ signalling, which exerts a putative anti-apoptotic influence. Moreover, other putative neuroprotective strategies have been proposed, based on stimulation by cells of the production of neurotrophic factors, including physical exercise, but also cellular and gene therapies. Interestingly, one of these factors, the brain derived neuronal factor (BDNF), has been recently extensively studied and it has been speculated that BDNF could actually have therapeutic interest (see Nagahara and Tuszynski 2011). However, experiments recently performed in an experimental model of Parkinson's disease using BDNF gene delivery do not verify its positive contribution (Decressac et al. 2011). Nevertheless, one has to consider that BDNF is not the sole candidate as a possible contributor to brain homeostasis since more than 50 different trophic factors such as GDNF or NGF, for example, have been identified in the brain. Moreover, such a result does not mean that BDNF gene delivery will not be efficient in another neurodegenerative disease although clinical trials have also failed for ALS (see Nagahara and Tuszynski 2011). Interestingly, the peptide mixture cerebrolysin, which exhibits neurotrophic effects, was shown in the animal model to reduce $\mathrm{A} \beta$ production and deposit (Rockenstein et al. 2006).

Compounds acting to reduce glutamatergic transmission could also be of interest regarding the possible role of excitotoxicity in neurodegenerative diseases. Animal models provide evidence of a possible positive influence of compounds acting on the glutamatergic receptor of the mGluR3 subtypes, which are known to decrease glutamate release and of a possible interest in cannabinoid agents acting as CB1 receptor antagonists. But in this last case, data are sometimes controversial in the literature.

Regarding a form of possible pre-disposition to neurodegenerative diseases due to early events in the life as suggested for Parkinson's disease (see Mabandla and Russell 2010) the question thus arises as to whether potential interventions which may prevent chronic intense stress consequences in juveniles, might provide some forms of neuroprotection strategies.

Last but not least, a therapeutical approach based on immunotherapy (active or passive immunotherapy) such as for example in Alzheimer's disease with a view to reducing brain amyloid deposits, has been investigated, as has also the use of amyloid- $\beta$ modulating agents such as $\beta$ - or $\gamma$-secretase inhibitors (such as CHF5074) or amyloid $\beta$-degrading agents such as neprilysin (Meilandt et al. 2009), as well as the administration of statins to limit cholesterol deposits (Sierra et al. 2011) or $\beta$-sheet breakers. Similarly, the ubiquitin-proteasome system could obviously represent a new target for developing novel therapeutics not only for neurodegenerative diseases but also immune-inflammatory disorders or even infectious diseases (see Bedford et al. 2011). The stimulation of cell survival pathways involving for example Akt signalling, PACAP stimulation (Reglodi et al. 2011), PARP modulation, NFk-B or ERK inhibition, could also represent an alternative to apoptose inhibition. In the case of Parkinson's disease one can further speculate that LRRK2 inducedinhibition or induced-expression of the parkin, PINK1 or DJ-1, for example, could also contribute to the protection of the dopaminergic neurons.

Modulation of neuronal excitability through agents modulating $\mathrm{Na}^{+} / \mathrm{Ca}^{2+}$ transporters or ions chelators such as desferrioxamine as well as a different subset of neuropeptides like cortexin or cortagen should also be of interest in the protection of the brain against neurodegenerative diseases.

An idea recently developed is that physical exercise could be one of the factors reducing the risk of developing neurodegenerative disease. Of course the demonstration of such assumption in humans is rather difficult but convergent data have been obtained from animal studies. Speculation on possible mechanisms proposes that such activity will increase the brain expression of trophic factors. Interestingly such a concept was extended to protection against cognitive decline based on the fact that certain epidemiologic studies have emphasized a decreased incidence of Alzheimer's disease in individuals who have intense intellectual activity, good educational and rather high cultural levels. So, the concept of 


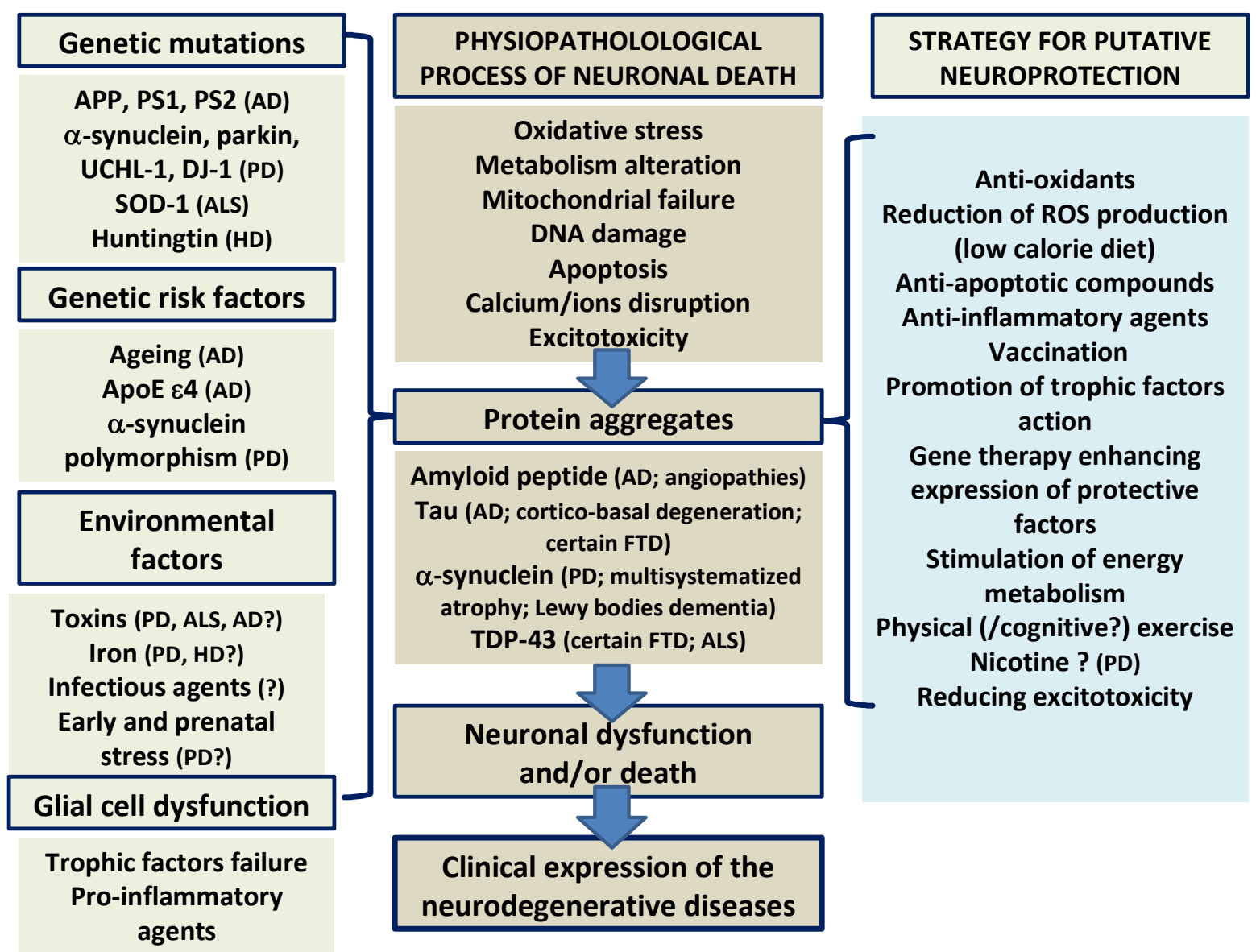

Fig. 1. Recapitulation of the processes which could be involved in the neurodegenerative diseases including genetic causal mutations and genetic risk factors possibly interfering with environmental factors to cause the diseases (first column). The second column presents possible mechanisms leading to protein aggregates and further to neuronal death, which contributes to clinical expression in neurodegenerative diseases. The last column presents putative strategies for active neuroprotection (AD: Alzheimer's disease; PD: Parkinson's disease; HD: Huntington's disease; ALS: amyotrophic lateral sclerosis; FTD: fronto-temporal dementia; TDP-43: TAR DNA-binding protein 43; ROS: reactive oxygen species).

"cognitive reserve" has been suggested, to represent a protective factor against dementia as well as physical activity.

Whatever strategy has been developed, however, at present the results are rather disappointing, except for riluzole, an anti-excitatory compound which was shown to be effective in ALS although the effects can be considered as limited. Does it mean that we are wrong and that we have to stop the search for putative neuroprotective drugs? The answer is of course certainly not. However, data could indicate that the concept has to be investigated in a less mechanistic direction or, alternatively, that we have to think in a more pertinent way in order to appreciate the neuroprotective effects in the clinic.

\section{CONCLUSIVE REMARKS}

In neuroscientific research, the field of neurodegenerative diseases is one of the most active in respect of both medical and associated social issues. Recent advances in the basic knowledge of such diseases have led to a re-evaluation of the pathological processes and to speculation about new therapeutical approaches (Fig. 1). These new therapies will include strategies based on better knowledge of the etiopathological processes and on more effective neuroprotection. Such mechanistic approaches to the diseases are still probably too global to be fully efficient in the short term. But we are fully convinced that new advances in the 
knowledge of the physiopathological mechanisms of the diseases including at molecular and genetic levels will further contribute to new therapeutic developments (see Nieoullon 2004). Research in this field is very active. Indeed, in the single field of Alzheimer's disease and related dementias, in 2010 nearly 100 medicines were being developed by biopharmaceutical research, but we are still waiting for definitive positive results. This is the reason why the research efforts from state governments and the $\mathrm{EU}$, together with the pharmaceutical industry, have to be reinforced, especially because, if we look at neurodegenerative diseases at least partly as proteinopathies it can be further speculated that curing one of the diseases could possibly contribute to a cure for the entire group of diseases related to a given proteinopathy. In this respect many biotech companies actively collaborate for example in the development of novel therapies based on the production of antibodies to modify the misfolding of proteins in Parkinson's and Alzheimer's diseases.

\section{REFERENCES}

Abou-Sleiman PM, Hanna MG, Wood NW. Genetic association studies of complex neurological diseases. J Neurol Neurosurg Psychiat. 77: 1302-1304, 2006.

Akwa Y, Allain H, Bentue-Ferrer D, Berr C, Bordet $\mathrm{R}$, Geerts H, Nieoullon A, Onteniente B, Vercelletto M. Neuroprotection and neurodegenerative diseases: from biology to clinical practice. Alzheimer Dis Assoc Disord. 19: 226-239, 2005.

Andlin-Sobocki P, Jönsson B, Wittchen H-U, Olesen J. Costs of disorders of the brain in Europe. Eur J Neurol. 12 (Suppl. 1): 1-27, 2005.

Ardley HC, Scott GB, Rose SA, Tan NG, Robinson PA. UCH-L1 aggresome formation in response to proteasome impairment indicates a role in inclusion formation in Parkinson's disease. J Neurochem. 90: 379-391, 2004.

Barlow BK, Cory-Slechta DA, Richfield EK, Thiruchelvam M. The gestational environment and Parkinson's disease: evidence for neurodevelopmental origins of a neurodegenerative disorder. Reprod Toxicol. 23: 457-470, 2007.

Barsukova AG, Bourdette D, Forte M. Mitochondrial calcium and its regulation in neurodegeneration induced by oxidative stress. Eur J Neurosci. 10: 437-447, 2011.
Bedford L, Lowe J, Dick LR, Mayer RJ, Brownell JE. Ubiquitin-like protein conjugation and the ubiquitin-proteasome system as drug targets. Nature Rev Drug Discovery. 10: 29-46, 2011.

Berger Z, Davies JE, Luo S, Pasco MY, Majoul I, O'Kane CJ, Rubinsztein DC. Deleterious and protective properties of an aggregate-prone protein with a polyalanine expansion. Hum Mol Genet. 15: 453-465, 2006.

Berger Z, Smith KA, Lavole MJ. Membrane localization of LRRK2 is associated with increased formation of the highly active LRRK2 dimer and changes in its phosphorylation. Biochemistry. 49: 5511-5523, 2010.

Brochard V, Combadiere B, Prigent A, Laouar Y, Perrin A, Beray-Berthat V, Bonduelle O, Alvarez-Fischer D, Callebert J, Launay JM, Duyckaerts C, Flavell RA et al. Infiltration of $\mathrm{CD}^{+}$lymphocytes into the brain contributes to neurodegeneration in a mouse model of Parkinson's disease. J Clin Invest. 119: 182-192, 2009.

Cicchetti F, Drouin-Ouellet J, Gross RE. Environmental toxins and Parkinson's disease: what have we learned from pesticide-induced animal models? Trends Pharmacol Sci. 30: 475-483, 2009.

Citron M. Strategies for disease modification in Alzheimer's disease. Nature Rev Neurosci. 5: 677-685, 2004.

Corti O, Fournier M, Brice A. Neurodegeneration in Parkinson's disease: Genetics enlightens physiopathology. J Neural Transm Suppl. 73: 215-221, 2009.

Decressac M, Ulusoy A, Mattsson B, Georgievska B, Romero-Ramos M, Kirik D, Björklund A. GDNF fails to exert neuroprotection in a rat (alpha)-synuclein model of Parkinson's disease. Brain. 134: 2302-2311, 2011.

Derkinderen P. Classification of neurodegenerative diseases: things are getting complicated (in French). La Lettre du Neurologue. 13: 68, 2009.

Dick FD. Parkinson's disease and pesticide exposures. Br Med Bull. 79-80: 219-231, 2006.

Djaldetti R, Lev N, Melamed E. Neuroprotection in progressive brain disorders. Isr Med Assoc J. 5: 576-580, 2003.

Friedman DB, Johnson TE. A mutation in the age-1 gene in Caenorhabditis elegans lengthens life and reduces hermaphrodite fertility. Genetics. 118: 75-86, 1988.

Gianaros PJ, Jennings JR, Sheu LK, Greer PJ, Kuller LH, Matthews KA. Prospective reports of chronic life stress predict decreased grey matter volume in the hippocampus. Neuroimage. 35: 795-803, 2007. 
Hampel H, Franck R, Broich K, Teipel SJ, Katz RG, Hardy J, Herholz K, Bokde ALW, Jessen F, Hoessler YC, Sanhai WR, Zetterberg H et al. Biomarkers for Alzheimer's disease: Academic, industry and regulatory perspectives. Nature Rev Drug Discov. 9: 560-574, 2010.

Hirsch EC, Hunot S. Neuroinflammation in Parkinson's disease: a target for neuroprotection? Lancet Neurol. 8: 382-397, 2009.

Holtzman DM, Bales KR, Tenkova T, Fagan AM, Parsadanian M, Sartorius LJ, Mackey B, Olney J, McKeel D, Wozniak D, Paul SM. Apolipoprotein $\mathrm{E}$ isoform-dependent amyloid deposition and neuritic degeneration in a mouse model of Alzheimer's disease. Proc Natl Acad Sci USA. 97: 2892-2897, 2000.

Hunter RL, Bing G. Agonism of peroxisome proliferator receptor-gamma may have therapeutic potential for neuroinflammation and Parkinson's disease. Curr Neuropharmacol. 5: 35-46, 2007.

Josephs KA. Frontotemporal dementia and related disorders: Deciphering the enigma. Ann Neurol. 64: 4-14, 2008.

Kristian T, Balan I, Schuh R, Onken M. Mitochondrial dysfunction and nicotinamide dinucleotide catabolism as mechanisms of cell death and promising targets for neuroprotection. J Neurosci Res. 10: 1002-1016, 2011.

Labrande C, Velly L, Canolle B, Guillet B, Masmejean F, Nieoullon A, Pisano P. Neuroprotecive effects of tacrolimus (FK506) in a model of ischemic cortical cell cultures: Role of glutamate uptake and FK506 binding protein 12 kDa. Neuroscience. 137: 231-239, 2006.

Llorente R, Gallardo ML, Berzal AL, Prada C, Garcia-Segura LM, Viveros MP. Early maternal deprivation in rats induces gender-dependent effects on developing hippocampal and cerebellar cells. Int J Dev Neurosci. 27: 233-241, 2009.

Mabandla MV, Russell VA. Voluntary exercise reduces the neurotoxic effects of 6-hydroxydopamine in maternally separated rats. Behav Brain Res. 211: 16-22, 2010.

Marais L, Van Rensburg SJ, Van Zyl JM, Stein DJ, Daniels WMU. Maternal separation of rat pups increases the risk of developing depressive-like behavior after subsequent chronic stress by altering corticosterone and neurotrophin levels in the hippocampus. Neurosci Res. 61: 106-112, 2008.

Meilandt WJ, Cisse M, Ho K, Esposito LA, Scearce-Levie K, Cheng IH, Yu GQ, Mucke L. Neprilysin overexpression inhibits plaque formation but fails to reduce pathogenic $A \beta$ oligomers and associated cognitive deficits in human amyloid precursor protein transgenic mice. J Neurosci. 18: 1977-1986, 2009.

Moisan F, Spinosi J, Dupupet JL, Delabre L, Mazurie JL, Goldberg M, Imbernon E, Tzourio C, Elbaz A. The relation between type of farming and prevalence of Parkinson's disease agricultural workers in five French districts. Mov Disord. 26: 271-279, 2011.

Nagahara AH, Tuszynski MH. Potential uses of BDNF in neurological and psychiatric disorders. Nature Rev Drug Discov. 10: 209-219, 2011.

Neumann M, Sampathu DM, Kwong LK, Truax AC, Micsenyi MC, Chou TT, Bruce J, Schuck T, Grossman M, Clark CM, McCluskey LF, Miller $\mathrm{BL}$ et al. Ubiquinated TDP-43 in fronto-temporal lobar degeneration and amyotrophic lateral sclerosis. Science. 314: 130-133, 2006.

Nieoullon A. Alzheimer's disease: neurobiological advances supporting proposals for new therapeutical approaches. J Appl Biomed. 2: 123-130, 2004.

Ozawa T, Healy DG, Abou-Sleiman PM, Ahmadi KR, Quinn N, Lees AJ, Shaw K, Wullner U, Berciano J, Moller JC, Kamm C, Burk K et al. The $\alpha$-synuclein gene in multiple system atrophy. J Neurol Neurosurg Psychiatry. 77: 464-467, 2006.

Reglodi D, Kiss P, Lubics A, Tamas A. Review on the protective effects of PACAP in models of neurodegenerative diseases in vitro and in vivo. Curr Pharm Des. 17: 962-972, 2011.

Rockenstein E, Torrance M, Mante M, Adame A, Paulino A, Rose JB, Crews L, Moessler H, Masliah E. Cerebrolysin decreases amyloid- $\beta$ production by regulating amyloid protein precursor maturation in a transgenic model of Alzheimer's disease. J Neurosci Res. 15: 1252-1261, 2006.

Sierra S, Ramos MC, Molina P, Esteo C, Vazquez JA, Burgos JS. Statins as neuroprotectants: a comparative in vitro study of lipophilicity, blood-brain-barrier penetration, lowering of brain cholesterol, and decrease of neuron cell death. J Alzheimers Dis. 23: 307-318, 2011.

Sleegers K, Cruts M, Van Broeckhoven C. Molecular pathways of frontotemporal degeneration. Ann Rev Neurosci. 33: 71-88, 2010.

Spivey JM, Shumake J, Colorado RA, ConejoJimenez N, Gonzales-Pardo H, Gonzales-Lima F. Adolescent female rats are more resistant than males to the effects of early stress on the prefrontal cortex and impulsive behavior. Dev Psychobiol. 51: 277-288, 2009.

Tiwari-Woodruff S, Morales LB, Lee R, Voskuhl RR. Differential neuroprotective and antiinflammatory effects of estrogen receptor (ER) $\alpha$ 
and ER $\beta$ ligand treatment. Proc Natl Acad Sci USA. 104: 14813-14818, 2007.

Vajda FJ. Neuroprotection and neurodegenerative disease. J Clin Neurosci. 9: 4-8, 2002.
Velly L, Guillet B, Masmejean F, Nieoullon A, Bruder N, Gouin FM, Pisano P. Neuroprotective effects of propofol in a model of ischemia cortical cell cultures. Role of glutamate and its transporters. Anesthesiology. 99: 368-375, 2003. 